\title{
The effect of ranibizumab and aflibercept treatment on the prevalence of outer retinal tubulation and its influence on retreatment in neovascular age-related macular degeneration
}

\author{
Attila Kovacs ${ }^{1}$, Timea Kiss ${ }^{1}$, Ferenc Rarosi ${ }^{2}$, Gabor M. Somfai ${ }^{3,4}$, Andrea Facsko ${ }^{1}$ and Rozsa Degi ${ }^{*}$
}

\begin{abstract}
Background: We aimed to analyze the differences in the prevalence of outer retinal tubulation (ORT) in neovascular age-related macular degeneration (AMD) treated with anti-vascular endothelial growth factor (antiVEGF) agents, either aflibercept or ranibizumab. Our further aim was to examine the changes in the frequency of injections of ranibizumab before and after ORT appearance.

Methods: Two hundred thirty six eyes of 230 patients were included in the study (184 eyes treated with ranibizumab by pro re nata regimen (PRN), 52 eyes with aflibercept bimonthly) and followed for 6-24 months. Using optical coherence tomography (OCT), the first appearance of ORT was documented, and fixed time point evaluations were also made every six months to determine the existence of ORT. The number of injections, the presence or absence of subretinal hyperreflective material (SHRM) at treatment initiation and visual acuity were also noted.

Results: The survival analysis with Cox proportional hazard model showed no significant difference between the ranibizumab and aflibercept groups in relation to the development of ORT ( $p=0.79$, hazard ratio 0.92 ). In the PRN treated ranibizumab group the number of injections showed significant decrease after ORT development $(p=0.004)$. When SHRM was present at treatment initiation the chance of developing ORT was 2.75 and 11.14 times higher in the ranibizumab and aflibercept groups, respectively.

Conclusions: The prevalence of ORT increased over time independently from the chosen anti-VEGF drug. Our results suggest that upon the appearance of ORT a decrease in retreatments can be expected.
\end{abstract}

Keywords: Outer retinal tubulation, Prevalence, Anti-VEGF, Aflibercept, Retreatment, Subretinal hyperreflective material

\section{Background}

Outer retinal tubulation (ORT) is a spectral-domain optical coherence tomography (SD-OCT) biomarker [1], first described by Zweifel et al. [2]. They defined ORTs as hyporeflective, branching tubular structures with hyperreflective borders within the outer nuclear layer of the retina [2]. The "en face" OCT technique can help map these

\footnotetext{
* Correspondence: degirozsa57@gmail.com

1 Department of Ophthalmology, Faculty of Medicine, University of Szeged,

10-11 Koranyi fasor, Szeged 6720, Hungary

Full list of author information is available at the end of the article
}

branching networks [3]. ORTs have been observed in many retinal diseases, including exudative age-related macular degeneration (AMD) [2]. Based on histological reports, the border of the outer retinal tubulation consists of photoreceptor inner segment mitochondria and external limiting membrane (ELM), with fluid and photoreceptor outer segments being potentially present in the ovoid hyporeflective lumen of the ORT [4-6]. Adaptive optics scanning laser ophthalmoscopy findings are in correlation with histology reports and show lack of ORT cone

(c) The Author(s). 2018 Open Access This article is distributed under the terms of the Creative Commons Attribution 4.0 International License (http://creativecommons.org/licenses/by/4.0/), which permits unrestricted use, distribution, and reproduction in any medium, provided you give appropriate credit to the original author(s) and the source, provide a link to the Creative Commons license, and indicate if changes were made. The Creative Commons Public Domain Dedication waiver (http://creativecommons.org/publicdomain/zero/1.0/) applies to the data made available in this article, unless otherwise stated. 
reflectivity which can be due to the loss of cone outer segments and subsequent retinal remodeling [7].

Schaal et al. classified outer retinal tubulations as either open (incomplete closure with curving external limiting membrane at the ends, horizontally elongated shape in cross-section) or closed (completely encircled, oval shape in cross-section) ORTs [4].

ORT can be mistaken for intraretinal cysts, or subretinal fluid but with the recognition of its hyperreflective border and special occurrence in the outer nuclear layer these mistakes can be reduced, leading to a reduction in the rate of anti-VEGF overtreatment in exudative AMD [2].

The ORT prevalence in exudative AMD is low at the time of first diagnosis but over time during anti-VEGF therapy its prevalence increases $[8,9]$. The importance of ORT as an OCT biomarker for photoreceptor degeneration is due to its connection with reduced visual acuity $[1,8-10]$.

It has been also reported that ORTs develop above areas of subretinal hyperreflective material (SHRM) or atrophy $[8,9]$. SHRM is a medium- to hyperreflective mass between the neurosensory retinal layers and retinal pigment epithelium on OCT [11]. It usually represents either a type II choroidal neovascular complex or is the consequence of an active choroidal neovascularisation, including subretinal haemorrhage and lipid or fluid exudation $[1,11,12]$.

The aim of the present study was to investigate the prevalence of ORTs in eyes with neovascular AMD undergoing treatment either with ranibizumab or aflibercept. Our further aim was to examine the changes in the frequency of injections before and after ORT appearance. We also assessed the presence of subretinal hyperreflective material and its relationship with ORT.

\section{Methods}

\section{Ethics, consent}

This retrospective study was performed at the Medical Retina Unit of the Ophthalmology Department of University of Szeged, in Hungary. The study was approved by the Institutional Review Board of University of Szeged Albert Szent-Györgyi Clinical Centre (reference number: 3650$)$ and was in accordance with the ethical standards of the Declaration of Helsinki. Since this was a retrospective review of patient data, informed consent was not required. The need for a consent was formally waived by the ethics committee, and this was also in line with the national regulations.

\section{Patients}

Treatment-naïve exudative AMD patients were enrolled in the study. For the ranibizumab group enrollment took place between October 2014 to April 2016 while patients in the aflibercept group were enrolled between April 2015 to April 2016.
All patients were over 50 years of age, the mean follow-up period was 16.3 months and 9.2 months (range 6-24 months and 6-12 months) in the ranibizumab and aflibercept groups, respectively.

During each visit a comprehensive ophthalmic examination was carried out including best-corrected visual acuity (BCVA, Early Treatment Diabetic Retinopathy Study (ETDRS) score) assessment, slit-lamp biomicroscopy, dilated funduscopy and SD-OCT examination of the retina (Heidelberg Spectralis, Heidelberg Engineering, Heidelberg, Germany). Eyes with poor quality SD-OCT scans (Q index below 20) or with poor compliance were excluded from the study (14 eyes from the ranibizumab and 3 eyes from the aflibercept group).

Treatment regimen for both ranibizumab $(0.5 \mathrm{mg})$ and aflibercept $(2 \mathrm{mg})$ started with 3 monthly injections. After this initiation phase the ranibizumab group was treated by a pro re nata (PRN) regimen with follow-up visits scheduled monthly. The retreatment criteria for ranibizumab patients consisted of any subretinal or intraretinal fluid on OCT, or new haemorrhage on funduscopy. In the aflibercept group follow-up after the loading phase was scheduled every two months, treatment was given at each follow-up. The above regimens were in accordance with the available treatment guidelines in Hungary at the time of the study.

For SD-OCT imaging a pattern size of $5.8 \times 5.8 \mathrm{~mm}$, $20^{\circ} \times 20^{\circ}$ was applied with $25 \mathrm{~B}$-scans, using the "follow-up" mode. By manual review of the scan volumes we determined the first appearance of the ORT in both groups. We also assessed the presence of ORT at fixed time points at baseline, month 6 and 12 in both groups and at months 18 and 24 in the ranibizumab group. Images were assessed by two independent retina specialists, in case of incongruity the images were referred to a third retina specialist to make a decision. During the evaluation of OCT scans we did not differentiate between the above described open (incomplete hyperreflective ring) and closed (complete hyperreflective ring) forms of ORT according to Schaal [4]. Thus, both types of ORT detected on the images were considered an ORT positive case. The criterion of ORT was a hyperreflective ovoid-elongated structure in the outer nuclear layer of the retina with lower reflective content (Fig. 1).

The readers also identified the presence or absence of subretinal hyperreflective material on SD-OCT images at treatment initiation. The criterion for SHRM was a medium- to hyperreflective mass between the neurosensory retinal layers and retinal pigment epithelium, as described by Keane et al. [11] (Fig. 1).

\section{Statistical methods}

The BCVA was compared across the two groups using the Mann-Whitney U-test. The survival analysis for 


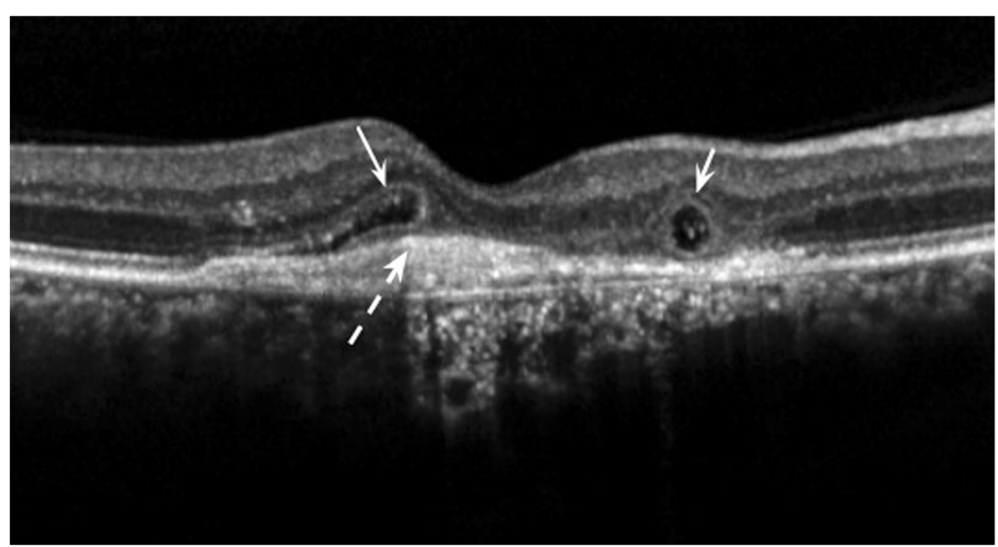

Fig. 1 Outer retinal tubulations and subretinal hyperreflective material on an SD-OCT single B-scan. Open and closed ORTs in cross section (left and right solid arrows) above subretinal hyperreflective material (dash arrow). The definition of ORT was a hyperreflective ovoid-elongated structure in the outer nuclear layer of the retina with lower reflective content

ORT development was analyzed by a Cox proportional hazard model. We analyzed the correlation between the presence of SHRM at treatment initiation with the development of ORT by Chi-square test and calculated relative risks. Where zeros were involved for the computation of relative risk, 0.5 was added to all cells, according to the previous suggestions [13].

The injection rate was calculated only in the PRN treated ranibizumab group due to the fixed 2 month therapeutic regimen of aflibercept. We assessed the injection rate only before and after the appearance of outer retinal tubulation and compared using the the Mann-Whitney U-test. In order to correct bias due to the unequal follow-up time (some patients had a higher number of injections due to the longer follow-up), we divided the follow-up time with the number of injections and calculated with monthly injections.

A $p$-value of $p<0.05$ was taken as statistically significant. For the analyses the IBM SPSS Software (Version 22) was used.

\section{Results}

In the ranibizumab group we evaluated 184 eyes of 179 patients, with a median age of 74 years (range 51 to 88), while in the aflibercept group there were 52 eyes of 51 patients with a median age of 75 years (range 58 to 87).

The mean baseline best corrected visual acuities in the two groups were (mean \pm SD) $59.16 \pm 13.9$ (median 61) and $53.96 \pm 13.54$ (median 55.5) ETDRS letters in the ranibizumab and aflibercept group, respectively. There was no significant difference between the two groups (Mann-Whitney U-test $p=0.083$ ). The BCVA at the end of the follow-up was $57.19 \pm 20.19$ (median 63) and $59.46 \pm 15.54$ (median 64) ETDRS letters in the ranibizumab and aflibercept group, respectively. There was no significant difference between the two groups (Mann-Whitney U-test $p=0.69$ ).

Table 1 shows the number of eyes during the follow-up in the two groups. The number of eyes was reduced over time due to gradual enrollment in the study, thereby not every patient reached the same follow-up time. In the ranibizumab group outer retinal tubulation was observed in $17.4 \%$ of cases at baseline, in $33.7 \%$ of cases at month 6 , in $45.3 \%$ of cases at month 12 , and in $55.3 \%$ and in $60.8 \%$ of cases at months 18 and 24, respectively. The ORT prevalence in the aflibercept group was $23.1 \%$ at baseline, $40.4 \%$ at month 6 , and $50 \%$ at month 12 .

The survival analysis showed no significant difference between the ranibizumab and aflibercept treated groups in terms of ORT development. $(p=0.79$, hazard ratio 0.92 , 95\% confidence interval 0.500-1.693) (Fig. 2).

The injection rates showed that the mean injection number per month before the ORT appearance was $0.37 \pm 0.17$ while after the ORT development it decreased to $0.21 \pm 0.17$ (Mann-Whitney U-test $p=0.004$ ).

The presence of subretinal hyperreflective material at treatment initiation in the two subgroups was $75.5 \%$ in the ranibizumab, and $80.8 \%$ in the aflibercept group. In

Table 1 Number of eyes reaching the follow-up in the ranibizumab and aflibercept treated groups

\begin{tabular}{lll}
\hline Time point & $\begin{array}{l}\text { Ranibizumab } \\
n \text { (eyes) }\end{array}$ & $\begin{array}{l}\text { Aflibercept } \\
n \text { (eyes) }\end{array}$ \\
\hline Baseline & 184 & 52 \\
at 6 months & 184 & 52 \\
at 12 months & 161 & 28 \\
at 18 months & 103 & 0 \\
at 24 months & 51 & 0 \\
\hline
\end{tabular}

Table legend: The column with " $\mathrm{n}$ " corresponds to the number of eyes reaching the follow-up 


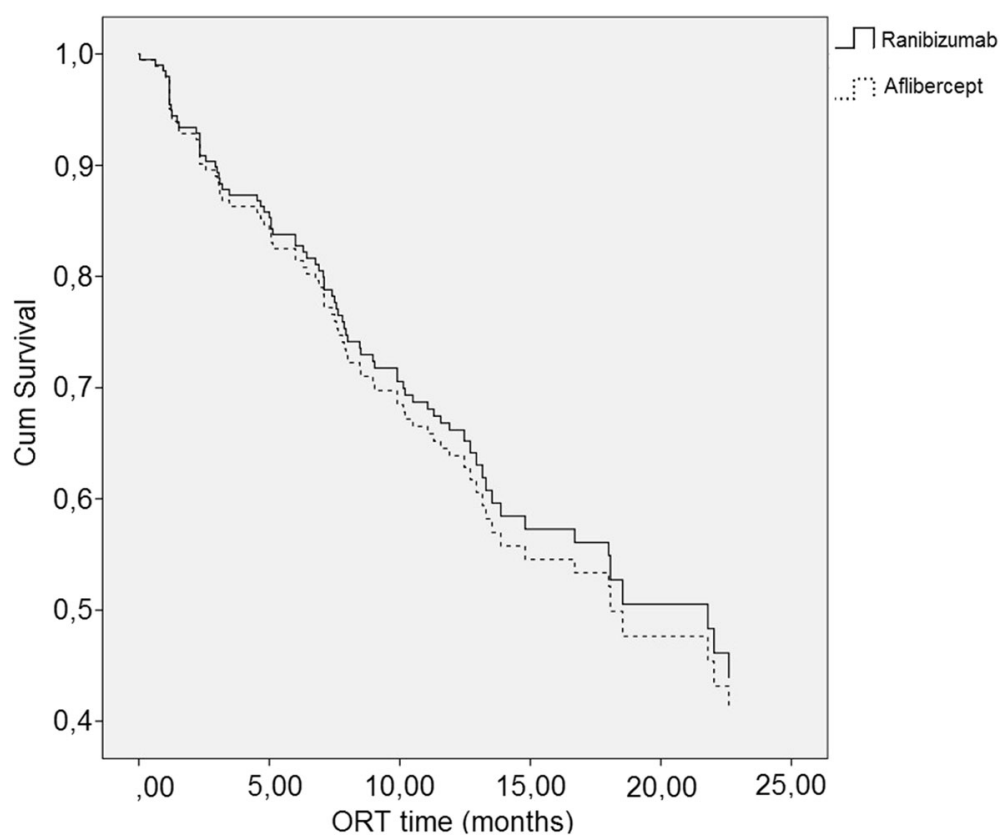

Fig. 2 Cox proportional hazard model of ORT survival in the ranibizumab and aflibercept groups

the ranibizumab treated group ORT developed in 85 eyes of 139 eyes with SHRM (61.15\%), while without SHRM (45 eyes) ORTs were found merely in 10 eyes $(22.2 \%)$ corresponding to a relative risk of 2.75. $(p<0.01)$. In the aflibercept treated group $55.81 \%$ of eyes with SHRM developed ORT (24 eyes of 43). No ORT developed in the eyes without SHRM (out of 9 eyes), consistent with a relative risk of $11.14(p<0.01)$.

\section{Discussion}

Our study found no significant difference between the ranibizumab and aflibercept treated groups according to ORT development. There was a statistically significant reduction in the frequency of injections in the ranibizumab treated group before and after ORT appearance. The presence of SHRM at treatment initiation as a biomarker had a statistically significant correlation with the development of ORT in both groups.

Zweifel first described outer retinal tubulation in 2009 [2]. Later the authors reported ORTs in various retinal disorders like neovascular AMD, geographic atrophy, polypoidal choroidal vasculopathy, non-AMD associated choroidal neovascularisation, and other degenerative retinal disorders (e.g. retinitis pigmentosa, gyrate atrophy, choroideremia, Stargardt disease, pattern dystrophy) [2, 3, 14-19].

The pathogenesis of ORT formation is still not completely clear, though recent reports helped a lot exploring it. Dolz-Marco et al. called the attention on the role of Müller cells in the pathomechanism of ORT development, namely the progressive photoreceptor damage that can result in Müller cell activation which thereby starts to produce glial fibrillary acidic protein, facilitating the formation of ORT [20]. Based on histological examinations it seems that the evolution of ORT starts with ELM and ellipsoid zone disruption [4]. ELM starts to scroll inward at its free edges, representing an initial form of ORT, leading to the development of a formed open ORT. With time the large, open ORTs split, their margins beginning to scroll ending in multiple smaller closed ORTs. During the scrolling process a downward displacement of adjacent inner nuclear layer and outer plexiform layer happens separating each ORT, and causing the appearance of microcystic lesions in the inner nuclear layer. The downward displacement of these layers might be due to the involvement of Müller cells in this scrolling/dragging process as Müller cells are contributing to the constitution of ELM with the inner segments of photoreceptors $[4,15,20,21]$.

Most of the studies in the field focused on exudative AMD and its relationship with ORT. From these reports it is known that ORT is an SD-OCT biomarker, the prevalence of which increases with time and is associated with decreased visual acuity $[1,8,9]$. It has been also reported that ORTs develop adjacent to areas of subretinal hyperreflective material or atrophy $[8,9]$. The differential diagnostic importance of outer retinal tubulation lies in the avoidance of overtreatment due to its similarity to intraretinal fluid $[2,8]$.

In the present study, we evaluated treatment-naïve exudative AMD patients treated with ranibizumab and 
aflibercept regarding the presence of outer retinal tubulation. Altogether 236 eyes were followed in both groups with no statistical difference between the baseline characteristics of the two groups considering age and BCVA.

The prevalence of ORT continuously increased during the follow-up period, in both groups. In the ranibizumab group its prevalence almost quadrupled at the 24-month follow-up, while there was a doubling in the aflibercept group in 12 months. It is important to note, that in the ranibizumab group the baseline prevalence was lower (17.4\% versus $23.1 \%)$. Dirani et al. found a similar increasing trend in their study starting with $2.5 \%$ at presentation, reaching $41.6 \%$ at 4 years of follow-up [8]. In our study the baseline ORT prevalence was higher compared to other reports $[8,9]$. The reason behind this could be the more advanced disease state at the time of presentation (due to the real life nature of our retrospective study) and a relative delay in therapy initiation due to country-specific financial difficulties. The poorer baseline BCVA in both groups also supports this idea.

There is one previous article known, describing ORT development in 24 non-treatment-naïve eyes, treated with aflibercept only after receiving at least 6 ranibizumab injection, reporting an initial 97\% ORT prevalence which later decreased to $75 \%$ [22].

To our knowledge, our study is the first to report results in treatment naïve patients treated with aflibercept and its connection with ORT development. The Cox proportional hazard model analysis suggested that there was no difference between the two in-label therapies ranibizumab and aflibercept in regard to the prevalence of outer retinal tubulation. Lee et al. in the Comparison of AMD Treatment Trials (CATT) study group evaluated the prevalence of ORTs in ranibizumab and bevacizumab treated neovascular AMD patients, and found no difference between the two drugs related to the prevalence of outer retinal tubulation [9].

In the present study we found a statistically significant difference in the monthly injection rate before and after the appearance of outer retinal tubulation in the ranibizumab treated group. Our results suggest that in patients who develop ORT a decrease in the retreatment rate can be expected which may be a very important clinical marker. Although we had a PRN regimen according to the Hungarian guidelines, Lee et al. found no difference between the fixed monthly regimen versus PRN regimen in regards to ORT development in patients treated either with ranibizumab or bevacizumab [9].

We found a statistically significant connection between ORT development and the presence of subretinal hyperreflective material at treatment initiation. When subretinal hyperreflective material was present the chance of developing ORT was 2.75., and 11.14 higher in the ranibizumab and aflibercept groups, respectively, in accordance with the results of Lee et al. in ranibizumab and bevacizumab treated patients [9].

Our findings, in concordance with the above mentioned study results suggest that ORT is independent of the chosen anti-VEGF drug or the dosing regimen of intravitreal anti-VEGF treatment. The appearance of ORT suggests that the clinicians can expect a decrease in the number of injections when following a pro re nata ranibizumab regimen. Our fixed bimonthly treatment with aflibercept did not allow us to analyze the injection rate before and after ORT development in this group. Our study also supports the previously reported higher prevalence of ORT development in the presence of subretinal hyperreflective material at treatment initiation [9].

There is a number of limitations of our study. Namely, the relatively small sample size in the aflibercept group compared to the ranibizumab group, along with the bimonthly follow-up in the aflibercept group. We believe that the number of subjects involved in both groups is comparable with other studies published in the field, while the bimonthly treatment regimen with aflibercept was fixed due to the country-specific guideline regulations. The decreasing number of eyes during the follow-up could also bias the analysis by including patients with increasing disease severity. However, we believe these factors were similar to those in similar studies available in the field. The strength of this report is the comparison of present in-label therapies, ranibizumab and aflibercept in exudative AMD patients in relation to ORT besides the evaluation of the injection rate in association with outer retinal tubulation. We used real life data that makes the study more relevant in the daily clinical practice.

\section{Conclusions}

The development of ORT could be a potential biomarker for the treatment prognosis in patients with wet AMD. Its clinical significance lies also in its similarity to activity-related intraretinal fluid. Further studies are needed to explore the nature and development of ORTs employing a comparable dosing and follow-up regimen of all three currently available anti-VEGF drugs ranibizumab, bevacizumab and aflibercept.

\section{Abbreviations \\ AMD: Age-related macular degeneration; anti-VEGF: Anti-vascular endothelial growth factor; BCVA: Best-corrected visual acuity; CATT: Comparison of AMD Treatment Trials; CNV: Choroidal neovascularisation; ELM: External limiting membrane; ETDRS: Early Treatment Diabetic Retinopathy Study; OCT: Optical coherence tomography; ORT: Outer retinal tubulation; PRN: Pro re nata; SD- OCT: Spectral-domain optical coherence tomography; SHRM: Subretinal hyperreflective material}

\section{Acknowledgments}

This research received no specific grant from any funding agency in the public, commercial, or not-for-profit sectors.

Funding

No funding was received for this research. 


\section{Availability of data and materials}

The datasets used and analyzed during the current study are available from the corresponding author on reasonable request.

All data generated or analyzed during this study are included in this published article [and its supplementary information files].

\section{Authors' contributions}

AK, RD made the conception and design of the study and analyzed the OCT images, in case of incongruity the final decision was made by AF. AK and TK collected and arranged the data (demographics, visual acuity, injection number and date). FR made the statistical analyzes and also the interpretation of data together with GMS. AK, TK drafted the manuscript. GMS was a major contributor editing it. AF, GMS and RD revised the manuscript. All authors read and approved the final manuscript.

\section{Ethics approval and consent to participate}

This study was approved by the the Human Investigation Review Board of the University of Szeged Albert Szent-Györgyi Clinical Centre (reference number: 3650) and was performed in accordance with the tenets of the Declaration of Helsinki. In the study we used a retrospective review of patient data; therefore consent was not collected from participants. The need for a consent was formally waived by the ethics committee, and this was also in line with the national regulations (a reference to the relevant legislation: 23/2002. (V. 9.) Eüm 20/Q. §).

\section{Consent for publication}

The attached OCT image is entirely unidentifiable and there are no details on individuals reported within the manuscript.

\section{Competing interests}

The authors declare that they have no competing interests.

\section{Publisher's Note}

Springer Nature remains neutral with regard to jurisdictional claims in published maps and institutional affiliations.

\section{Author details}

'Department of Ophthalmology, Faculty of Medicine, University of Szeged, 10-11 Koranyi fasor, Szeged 6720, Hungary. ${ }^{2}$ Department of Medical Physics and Informatics, Faculty of Medicine, University of Szeged, Szeged, Hungary. ${ }^{3}$ Augenzentrum Pallas Kliniken, Olten, Switzerland. ${ }^{4}$ Department of Ophthalmology, Faculty of Medicine, Semmelweis University, Budapest, Hungary.

\section{Received: 2 November 2017 Accepted: 26 October 2018}

\section{Published online: 14 November 2018}

\section{References}

1. Schmidt-Erfurth U, Waldstein SM. A paradigm shift in imaging biomarkers in neovascular age-related macular degeneration. Prog Retin Eye Res. 2016;50: 1-24. https://doi.org/10.1016/j.preteyeres.2015.07.007.

2. Zweifel SA, Engelbert M, Laud K, Margolis R, Spaide RF, Freund KB. Outer retinal tubulation: a novel optical coherence tomography finding. Arch Ophthalmol. 2009;127(12):1596-602. https://doi.org/10.1001/archophthalmol. 2009.326.

3. Wolff B, Matet A, Vasseur V, Sahel JA, Mauget-Faÿsse M. En face OCT imaging for the diagnosis of outer retinal tubulations in age-related macular degeneration. J Ophthalmol. 2012;2012:542417. https://doi.org/10.1155/ 2012/542417.

4. Schaal KB, Freund KB, Litts KM, Zhang Y, Messinger JD, Curcio CA. Outer retinal tubulation in advanced age-related macular degeneration: optical coherence tomographic findings correspond to histology. Retina. 2015;35(7): 1339-50. https://doi.org/10.1097//AE.0000000000000471.

5. Litts KM, Messinger JD, Freund KB, Zhang Y, Curcio CA. Inner segment remodeling and mitochondrial translocation in cone photoreceptors in agerelated macular degeneration with outer retinal Tubulation. Invest Ophthalmol Vis Sci. 2015;56(4):2243-53. https://doi.org/10.1167/iovs.14-15838.

6. Litts KM, Messinger JD, Dellatorre K, Yannuzzi LA, Freund KB, Curcio CA. Clinicopathological correlation of outer retinal tubulation in age-related macular degeneration. JAMA Ophthalmol. 2015;133(5):609-12. https://doi. org/10.1001/jamaophthalmol.2015.126.
7. Litts KM, Wang X, Clark ME, Owsley C, Freund KB, Curcio CA, Zhang Y. Exploring photoreceptor reflectivity through multimodal imaging of outer retinal tubulation in advanced age-related macular degeneration. Retina. 2017:37(5):978-88. https://doi.org/10.1097//AE.0000000000001265.

8. Dirani A, Gianniou C, Marchionno L, Decugis D, Mantel I. Incidence of outer retinal tubulation in ranibizumab-treated age-related macular degeneration. Retina. 2015;35(6):1166-72. https://doi.org/10.1097//AE.0000000000000439.

9. Lee JY, Folgar FA, Maguire MG, Ying GS, Toth CA, Martin DF, Jaffe GJ, CATT Research Group. Outer retinal tubulation in the comparison of age-related macular degeneration treatments trials (CATT). Ophthalmology. 2014; 121(12):2423-31. https://doi.org/10.1016/j.ophtha.2014.06.013.

10. Faria-Correia F, Barros-Pereira R, Queirós-Mendanha L, Fonseca S, Mendonça L, Falcão MS, Brandão E, Falcão-Reis F, Carneiro AM. Characterization of neovascular age-related macular degeneration patients with outer retinal tubulations. Ophthalmologica. 2013;229(3):147-51. https://doi.org/10.1159/ 000346854.

11. Keane PA, Patel PJ, Liakopoulos S, Heussen FM, Sadda SR, Tufail A. Evaluation of age-related macular degeneration with optical coherence tomography. Surv Ophthalmol. 2012;57(5):389-414. https://doi.org/10.1016/j. survophthal.2012.01.006.

12. Shah VP, Shah SA, Mrejen S, Freund KB. Subretinal hyperreflective exudation associated with neovascular age-related macular degeneration. Retina. 2014; 34(7):1281-8. https://doi.org/10.1097//AE.0000000000000166.

13. Agresti A. Categorical data analysis. 2nd ed. Gainesville: Wiley; 2002.

14. Hariri A, Nittala MG, Sadda SR. Outer retinal tubulation as a predictor of the enlargement amount of geographic atrophy in age-related macular degeneration. Ophthalmology. 2015;122(2):407-13. https://doi.org/10.1016/j. ophtha.2014.08.035.

15. Hua R, Liu L, Hu Y, Chen L. The occurrence and progression of outer retinal tubulation in Chinese patients after intravitreal injections of ranibizumab. Sci Rep. 2015;7(5):7661. https://doi.org/10.1038/srep07661.

16. Goldberg NR, Greenberg JP, Laud K, Tsang S, Freund KB. Outer retinal tubulation in degenerative retinal disorders. Retina. 2013;33(9):1871-6. https://doi.org/10.1097/IAE.0b013e318296b12f.

17. Iriyama A, Aihara Y, Yanagi Y. Outer retinal tubulation in inherited retinal degenerative disease. Retina. 2013;33(7):1462-5. https://doi.org/10.1097/IAE. ob013e31828221ae.

18. Giachetti Filho RG, Zacharias LC, Monteiro TV, Preti RC, Pimentel SG. Prevalence of outer retinal tubulation in eyes with choroidal neovascularization. Int J Retina Vitreous. 2016;2(1):6. https://doi.org/10.1186/ s40942-016-0029-8.

19. Jung JJ, Freund KB. Long-term follow-up of outer retinal tubulation documented by eye-tracked and en face spectral-domainoptical coherence tomography. Arch Ophthalmol. 2012;130(12):1618-9. https://doi.org/10. 1001/archophthalmol.2012.1902.

20. Dolz-Marco R, Litts KM, Tan ACS, Freund KB, Curcio CA. The evolution of outer retinal tubulation, a neurodegeneration and gliosis prominent in macular diseases. Ophthalmology. 2017;124(9):1353-67. https://doi.org/10. 1016/j.ophtha.2017.03.043

21. Preti RC, Govetto A, Filho RGA, Cabral Zacharias L, Gianotti Pimentel S, Takahashi WY, Monteiro MLR, Hubschman JP, Sarraf D. Optical coherence tomography analysis of outer retinal tubulations: sequential evolution and pathophysiological insights. Retina. 2018;38(8):1518-25. https://doi.org/10. 1097/IAE.0000000000001810.

22. Massamba N, Dirani A, Butel N, Fardeau C, Bodaghi B, Ingram A, Lehoang P. Evaluation of outer retinal tubulations in eyes switched from intravitreal ranibizumab to aflibercept for treatment of exudative age-related macular degeneration. Graefes Arch Clin Exp Ophthalmol. 2017;255(1):61-7. https:// doi.org/10.1007/s00417-016-3423-x. 